\title{
Grating Couplers in Ultrananocrystalline Diamond
}

\author{
Marie Verbist and Dries Van Thourhout \\ Photonics Research Group, INTEC-department, Ghent University - IMEC \\ Center for Nano- and Biophotonics (NB-Photonics), Ghent University \\ Ghent, Belgium \\ Email: marie.verbist@intec.ugent.be
}

\begin{abstract}
Grating couplers and waveguides were fabricated in a thin layer of ultrananocrystalline diamond with a focusedion-beam. The measured spectra were linked to simulations to estimate material-related propagation losses.
\end{abstract}

\section{INTRODUCTION}

The extreme qualities of diamond make it desirable for a wide range of applications. For integrated optical devices, this includes its wide transparancy range and high refractive index (2.4), but also exceptional hardness, thermal conductivity and a wide range of optically active defects. To exploit these properties, waveguides and coupling structures have to be developed.

\section{FABRICATION}

\section{A. Thin diamond layers}

Although thin diamond layers can be fabricated from bulk monocrystalline diamond [1], growing thin layers by chemical vapour deposition is more flexible and easier to scale [2]. Ultrananocrystalline layers of diamond are commercially available. We purchased a wafer from Advanced Diamond Technologies, Inc. It has a $<100>$ silicon wafer base with a thermal silicon oxide layer of $1 \mu \mathrm{m}$. The diamond layer was grown to a thickness of $330 \mathrm{~nm}$ and has surface roughness below $10 \mathrm{~nm}$ rms (Fig. 1).

\section{B. Focused Ion Beam etching}

As can be expected, the extreme properties of diamond pose fabrication challenges. We use a dual beam focused-ion-beam (FIB) because it offers both flexibility and a short development cycle. It has the known disadvantages of implanting ions and damaging the sidewalls and bottom, leading to additional optical losses [4]. Therefore, we protect the diamond surface by covering it with an alumininum-oxide layer of about $50 \mathrm{~nm}$.

1) Waveguides: A waveguide is formed by etching two trenches. As this is a long structure, it cannot be etched at once without compromising the resolution. Instead, it is necessary to etch part of the waveguide and move the sample stage to continue with the next part. This is called stitching. To this end, we have developed an automatic alignment procedure based on image recognition of alignment markers [3]. Fig. 2a show one stitch (only visible in the trenches) and two markers that were imaged for alignment.
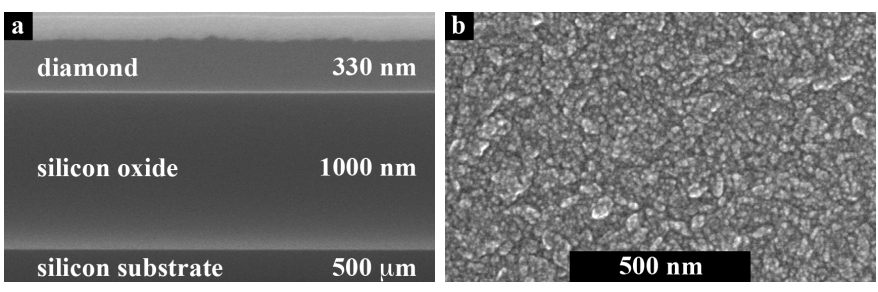

Fig. 1. (a) $330 \mathrm{~nm}$ of ultrananocrystalline diamond on $1 \mu \mathrm{m}$ of silicon oxide. (b) Surface roughness of the same thin diamond layer.
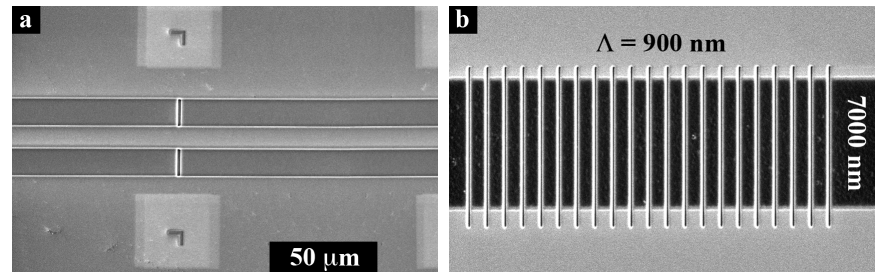

Fig. 2. (a) Part of a FIB-etched waveguide, showing one stitch and two alignment markers. (b) Ion image of FIB-etched grating coupler.

2) Grating couplers: Instead of etching rectangles that lead to the optimal fill factor, we use a line etch. This reduces the fraction of the grating coupler over which excess losses are introduced. Fig. $2 \mathrm{~b}$ shows an ion image which was taken immediately after etching the grating. The exact width of the lines - and thus the fill factor of the grating - is mainly determined by the ion current and the etch depth. It also depends on the quality of the beam focus, which has to be optimized carefully.

\section{Simulations}

Simulations in CAMFR, a full-vectorial solver based on eigen mode expansion, reveal the spectrum that a grating coupler with certain design parameters couples from optical fiber to waveguide/slab. The central wavelength and efficiency do not only depend on the grating period, but also on the exact refractive index, layer thicknesses, etch depth and fill factor. At a wavelength of $1550 \mathrm{~nm}$, we obtained an optimal efficiency of $39 \%$ for a grating period of $900 \mathrm{~nm}$, etch depth of $275 \mathrm{~nm}$ and a fill factor of $85 \%$. As FIB etched slits are not perfectly rectangular, and fill factor and etch depth are closely related, the simulations are repeated after fabrication, using parameters extracted from cross-sections. 

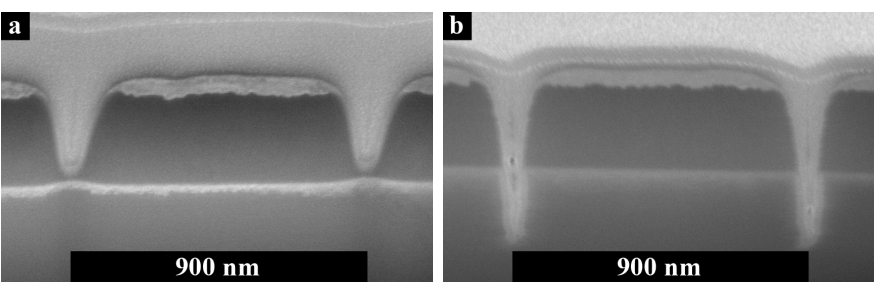

Fig. 3. The gratings of the experiment in the slab (a) have a lower fill factor en lower etch depth than the gratings of the waveguide experiment (b).

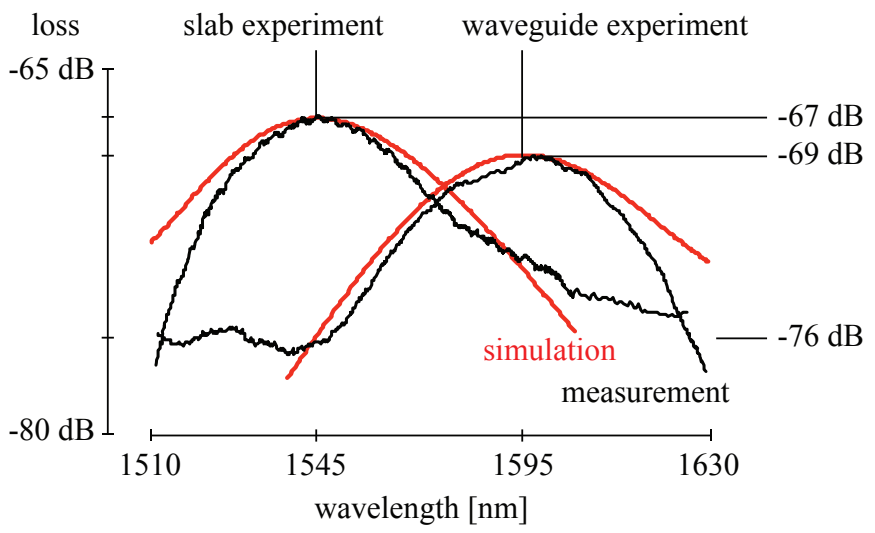

Fig. 4. The measured spectra (in black) show a wavelength shift of $45 \mathrm{~nm}$. The simulated spectra (in red) correspond well with the measurements.

\section{EXPERIMENTS}

Two experiments will be compared. For the first experiment, we etched two grating couplers in the slab at a distance of $500 \mu \mathrm{m}$. Without horizontal confinement, the light will diffract in the entire slab. For the second experiment, we started by etching a strip waveguide after which two grating couplers were added, again at a distance of $500 \mu \mathrm{m}$. It is important to note that both sets of grating couplers are not the same. Although they were etched using the same FIB parameters, a difference in beam focus caused a very distinct difference in fill factor and etch depth (Fig. 3). The measured spectra (Fig. 4) reveal this as a significant wavelength shift between both experiments $(45 \mathrm{~nm})$. The total losses are similar $(67$ $\mathrm{dB}$ and $69 \mathrm{~dB}$ ), but this is a coincidence as the various loss contributions are quite different.

These loss contributions can be divided into coupling losses and propagation losses (see table I). To estimate the coupling losses, we repeat the CAMFR simulations, based on the information from the cross-sections in Fig. 3. The central wavelength is fitted to the measurements (Fig. 4), which results in a loss per grating coupler of $4 \mathrm{~dB}$ and $11 \mathrm{~dB}$ respectively. As the grating couplers are $7 \mu \mathrm{m}$ wide and the fiber core is $10 \mu \mathrm{m}$ wide, we have to take into account an additional mode mismatch loss. This is not included in the (two-dimensional) CAMFR simulations. We estimate this loss to be about $3 \mathrm{~dB}$ per grating coupler. Finally, the coupling losses should also include FIB-induced losses. These are difficult to calculate, but we estimate them to be responsible for at least an additional $10 \mathrm{~dB}[4]$.
TABLE I

SUMMARY OF LOSS CONTRIBUTIONS

\begin{tabular}{lrrr}
\hline & slab & waveguide & \\
\hline Coupling Losses & & & \\
$\quad$ Grating coupler efficiency & $-8 \mathrm{~dB}$ & $-22 \mathrm{~dB}$ & simulated \\
Mode mismatch & $-6 \mathrm{~dB}$ & $-6 \mathrm{~dB}$ & calculated \\
FIB-related & $-10 \mathrm{~dB}$ & $-10 \mathrm{~dB}$ & estimated \\
Propagation Losses & & & \\
$\quad$ Diffraction & $-18 \mathrm{~dB}$ & $0 \mathrm{~dB}$ & calculated \\
Stitching \& sidewalls & $0 \mathrm{~dB}$ & $-6 \mathrm{~dB}$ & extracted \\
$\quad$ Scattering \& absorption & $\mathbf{- 2 5} \mathbf{~ d B}$ & $\mathbf{- 2 5} \mathbf{~ d B}$ & extracted \\
\hline Total loss & $-67 \mathrm{~dB}$ & $-69 \mathrm{~dB}$ & measured \\
\hline
\end{tabular}

The propagation losses differ for both experiments. In the slab experiment, we must take beam diffraction into account. Over a distance of $500 \mu \mathrm{m}$, we have calculated this to be responsable for a loss of $18 \mathrm{~dB}$. In the waveguide experiment, the stitching and sidewall roughness will cause additional losses, which are difficult to estimate/simulate. Comparing all previous loss contributions with the total measured loss, we still haven't accounted for $25 \mathrm{~dB}$ and $31 \mathrm{~dB}$ respectively. We contribute the difference of $6 \mathrm{~dB}$ to stitch/sidewall losses. We consider the remaining $25 \mathrm{~dB}$ or $\mathbf{5 0 d B} / \mathbf{m m}$ to be an upper limit for the propagation losses due to absorption and scattering in the ultrananocrystalline diamond.

\section{Conclusion}

We have simulated, fabricated and measured grating couplers in a thin layer of ultrananocrystalline diamond. We compared propagation in the slab with propagation in the waveguide. By estimating the various loss contributions we have extracted an upper limit for propagation losses due to material absorption and scattering of $50 \mathrm{~dB} / \mathrm{mm}$. To reach a more conclusive number, our future work will include cut-back measurements. By optimizing the grating couplers, measuring longer waveguides will become possible. We also plan to perform these measurements at shorter wavelengths.

\section{ACKNOWLEDGMENT}

This work was supported by the Institute for the Promotion of Science and Technology in Flanders (IWT) under a grant.

\section{REFERENCES}

[1] S. Castelletto, J. P. Harrison, L. Marseglia, A. C. Stanley-Clarke, B. C. Gibson, B. A. Fairchild, J. P. Hadden, Y. L. D. Ho, M. P. Hiscocks, K. Ganesan, S. T. Huntington, F. Ladouceur, A. D. Greentree, S. Prawer, J. L. O'Brien, and J. G. Rarity, "Diamond-based structures to collect and guide light," New Journal of Physics, vol. 13, p. 02502, Feb 2011.

[2] O. A. Williams, M. Nesladek, M. Daenen, S. Michaelson, A. Hoffman, E. Osawa, K. Haenen, and R. B. Jackman, "Growth, electronic properties and applications of nanodiamond," Diamond and Related Materials, vol. 17, pp. 1080-1088, 2008.

[3] M. Verbist and D. Van Thourhout, "Focused ion beam etching of thin diamond layers," Proceedings of the 2009 Annual Symposium of the IEEE Photonics Benelux Chapter, pp. 113-116, Nov 2009.

[4] J. Schrauwen, D. Van Thourhout, and R. Baets, "Focused-ion-beam fabricated vertical fiber couplers on silicon-on-insulator waveguides," $3 \mathrm{rd}$ IEEE International Conference on Group IV Photonics, pp. 101-103, 2006. 